In Cres. Vol. $5 N^{\circ} 1:$ pp. 37-48, 2014

\title{
ESTILO DE VIDA Y ESTADO NUTRICIONAL DEL ADULTO MAYOR DEL PUEBLO JOVEN LA UNIÓN, CHIMBOTE $^{*}$
}

\author{
LIFESTYLE AND NUTRITION STATUS OF OLDER YOUNG OF \\ PUEBLO JOVEN LA UNION, CHIMBOTE
}

\author{
Yosselyn Mirelly Pardo Robles ${ }^{1}$ \\ Yolanda Rodríguez Núñez ${ }^{2}$
}

\begin{abstract}
RESUMEN
El presente trabajo de investigación, de tipo cuantitativo, con diseño descriptivo, se realizó con el propósito de determinar el estilo de vida y el estado nutricional del adulto mayor del P.J. La Unión, sector 7, Chimbote, 2012. El universo muestral estuvo constituido por 171 adultos mayores de ambos sexos. Para la recolección de datos se aplicó tres instrumentos: la escala de estilo de vida, la ficha de valoración nutricional del adulto mayor y la tabla de valoración nutricional según índice de masa corporal (IMC) para adultos mayores ( $>60$ años). El análisis y el procesamiento de datos se realizaron en el Software SPSS versión 18.0, elaborándose tablas y gráficos simples y porcentuales. Se llegó a las siguientes conclusiones: La mayoría de adultos mayores del P.J. La Unión, sector 7, presenta un estilo de vida no saludable y un mínimo porcentaje tiene un estilo de vida saludable. En cuanto al estado nutricional de los adultos mayores, tenemos que, cerca de la mitad presenta un estado nutricional normal; casi la cuarta parte presenta sobrepeso; un porcentaje menor presenta delgadez y una mínima parte, obesidad. Los adultos mayores del P.J. La Unión, sector 7, con estilo de vida no saludable presentan en mayor porcentaje estado nutricional normal, sobrepeso, delgadez y obesidad, respectivamente; y los adultos con estilo de vida saludable presentan en mínimo porcentaje obesidad, sobrepeso normal y delgadez, respectivamente.
\end{abstract}

PALABRAS CLAVE: Estilo vida, estado nutricional, adulto mayor.

* Recibido: 28 de enero del 2014; aprobado: 7 de junio del 2014.

1 Estudiante de Enfermería del VIII ciclo. Escuela Profesional de Enfermería de la Universidad Católica Los Ángeles de Chimbote. Chimbote - Perú.

2 Doctora en Ciencias de Enfermería. Docente principal de la Universidad Católica Los Ángeles de Chimbote. Chimbote - Perú. 


\begin{abstract}
The present research, quantitative, with descriptive design was carried out to determine the lifestyle and nutritional status of the elderly P.J. La Union, Sector 7, Chimbote, 2012. The sampling universe consisted of 171 elderly of both sexes. For data collection, three instruments were applied: the scale of lifestyle Record nutritional assessment of the elderly and the table of nutritional value according to body mass index (BMI) for older adults ( $>60$ years). The analysis and data processing were performed in SPSS version 18.0 software, making simple tables and graphs and percentages, reaching the following conclusions: Most seniors from P.J. La Unión, Sector 7, have unhealthy life style and a small percentage have a healthy lifestyle. Regarding the nutritional status of older adults PJ La Union, Sector 7, we have, about half of the elderly have normal nutritional status, almost a quarter are overweight, and thinness presents a lower percentage and fraction obesity. Seniors P.J. La Union, Sector 7 with unhealthy lifestyle have a higher percentage nutritional status normal, overweight, obesity and thinness respectively and adults with healthy lifestyle presented in minimum percentage obesity, overweight, normal and thin respectively.
\end{abstract}

KEY WORDS: Life style, nutritional status, elderly.

\title{
INTRODUCCIÓN
}

Esta investigación se fundamenta en las bases conceptuales de estilo de vida y estado nutricional del adulto mayor, teniendo en cuenta que ambos son importantes para el mantenimiento de la salud del adulto mayor, siendo esta una población que día a día demanda más atención.

El estilo de vida es la forma de vida de las personas o de los grupos. A pesar de no existir una definición unánime, la mayoría de los autores define a los estilos de vida como un conjunto de patrones conductuales que una persona en concreto pone en práctica de manera consistente y mantenida en su vida cotidiana y que puede ser pertinente para el mantenimiento de su salud y puede estar en situación de riesgo para la enfermedad; es decir, posee repercusiones importantes para la salud. ${ }^{1}$

El estado nutricional del adulto mayor se ve afectado por las características del envejecimiento, que es un fenómeno complejo que abarca cambios moleculares celulares, fisiológicos. Los problemas de salud y la declinación fisiológica se desarrollan progresivamente y en parte se debe a los malos hábitos sanitarios mantenidos durante toda la vida. ${ }^{2}$

\section{PROBLEMA}

¿Cuál es el estilo de vida y estado nutricional del adulto mayor del Pueblo Joven La Unión, sector 7, Chimbote en el año 2012? 


\section{OBJETIVO GENERAL}

Describir el estilo de vida según el estado de nutrición del adulto mayor del P.J. La Unión, sector 7, Chimbote.

\section{OBJETIVOS ESPECÍFICOS}

1. Valorar el estilo de vida del adulto mayor del P.J. La Unión, sector 7, Chimbote.

2. Identificar el estado nutricional del adulto mayor del P.J. La Unión, sector 7, Chimbote.

\section{JUSTIFICACIÓN}

La presente investigación es importante para el personal de salud que labora en el Puesto de Salud, CLASS La Unión, para que el usuario geriátrico reciba una atención de calidad conociendo su problemática, y en base a los resultados brindar una atención integral a las personas de este grupo etario en esta comunidad y reducir problemas referentes a su estado nutricional y estilo de vida.

Para el profesional de Enfermería esta investigación es útil porque contribuirá con conocimientos acerca del estilo de vida y de la situación nutricional del adulto mayor y desde una perspectiva educativa, preventiva, curativa y de rehabilitación, fortalecerá su rol de liderazgo dentro del equipo de salud, permitiéndole implementar programas preventivos promocionales para fomentar un estilo de vida saludable y asegurar un estado nutricional adecuado de los adultos mayores.

Así también, esta investigación permitirá que las instituciones formadoras de profesionales de la salud, como la Universidad Católica Los Ángeles de Chimbote, socialicen los resultados y que desde los primeros ciclos se incorporen contenidos sobre estilo de vida, así como un adecuado estado nutricional, para garantizar la formación de profesionales con una visión preventiva- promocional hacia la población adulta mayor.

Para las instituciones de formación profesional de la salud, los resultados de la investigación contribuirán en la ampliación de los contenidos curriculares referentes a la salud del adulto mayor, además de servir como fuente bibliográfica o de referencia, que permita desarrollar futuros trabajos de investigación similares, buscando alternativas de solución para mejorar el estado nutricional del adulto mayor.

Finalmente, el presente estudio servirá de marco referencial de conocimientos para estudiantes y en futuros trabajos de investigación. 


\section{METODOLOGÍA}

Este estudio es de tipo cuantitativo, de corte transversal, nivel aplicati$\mathrm{vo}^{3,4}$, descriptivo ${ }^{4}$. La población está conformada por 171 personas adultas mayores que residen en el P.J La Unión, sector, 7 del distrito de Chimbote, provincia del Santa, departamento de Ancash, utilizando los siguientes criterios de inclusión: Adulto mayor que resida más de 3 años en el P.J. La Unión, Sector 7, Chimbote; adulto mayor de ambos sexos; adulto joven que acepte participar en el estudio. Todos fueron seleccionados aleatoriamente, con un nivel de confianza del $95 \%$ y un margen de error permisible del $5 \%$.

Para la recolección de datos se aplicó la técnica de la entrevista, utilizando tres instrumentos: la escala del estilo de vida elaborado por Walker, Sechrist y Pender Delgado; según Reyna y Díaz, las tablas de valoración nutricional antropométrica según índice de masa corporal para adultos mayores ( $>60$ años) del Ministerio de Salud, y la ficha de valoración nutricional del adulto mayor. Es un instrumento elaborado por la metodología del Departamento de Metodología de la Investigación, donde se evidencia el peso, la talla, el índice de masa corporal y el diagnostico nutricional de cada adulto mayor evaluado, los cuales se garantizaron en su validez y confiabilidad mediante la prueba de Alpha de Crombach y el método de división en mitades.

\section{RESULTADOS}

Tabla 01

ESTILO DE VIDA DEL ADULTO MAYOR. P.J.

LA UNIÓN, SECTOR VII, CHIMBOTE, 2012.

\begin{tabular}{lcc}
\hline Estilo de vida & Frecuencia & Porcentaje \\
\hline Saludable & 6 & 3,5 \\
No saludable & 165 & 96,5 \\
\hline Total & 171 & 100,0 \\
\hline
\end{tabular}

Fuente: Escala de estilo de vida elaborada por: Walker, Sechrist y Pender, modificado por: Díaz, R; Reyna, E; Delgado, R. aplicado aal adulto mayor. P.J. La Unión, sector 7, Chimbote, 2012. 
Tabla 02

ESTADO NUTRICIONAL DEL ADULTO MAYOR

\begin{tabular}{lcc}
\hline Estado & Frecuencia & Porcentaje \\
\hline Delgadez & 42 & 24,6 \\
Normal & 66 & 38,6 \\
Sobrepeso & 48 & 28,1 \\
Obesidad & 15 & 8,8 \\
\hline
\end{tabular}

Fuente; Ficha de valoración del adulto mayor y tabla de valoración nutricional antropométrica según índice de masa corporal (IMC) del adulto mayores ( $>60$ años). Ministerio de salud (MINSA) aplicado a los adultos mayores que residen en el P.J. La Unión, Sector 7, Chimbote, 2012.

Tabla 03

ESTILO DE VIDA Y ESTADO NUTRICIONAL DEL ADULTO MAYOR

\begin{tabular}{lrrrrrrrrrr}
\hline \multirow{2}{*}{ Estilo de vida } & \multicolumn{2}{c}{ Delgadez } & \multicolumn{2}{c}{ Normal } & \multicolumn{2}{c}{ Sobrepeso } & \multicolumn{2}{c}{ Obesidad } & \multicolumn{2}{c}{ Total } \\
\cline { 2 - 12 } & $\mathbf{N}$ & $\mathbf{\%}$ & $\mathbf{N}$ & $\mathbf{\%}$ & $\mathbf{N}$ & $\boldsymbol{\%}$ & $\mathbf{N}$ & $\mathbf{\%}$ & $\mathbf{N}$ & $\mathbf{\%}$ \\
\hline Saludable & 1 & 0,6 & 2 & 1,2 & 2 & 1,2 & 1 & 0,6 & 6 & 3,5 \\
No Saludable & 41 & 24,0 & 64 & 37,4 & 46 & 26,9 & 14 & 8,2 & 165 & 96,5 \\
Total & 42 & 24,5 & 66 & 38,6 & 48 & 28,1 & 15 & 8,8 & 171 & 100,0 \\
\hline
\end{tabular}

Fuente: Escala de estilo de vida elaborada por Wálker, Sechrist y Pender, modificado por Díaz, R; Reyna E; Delgado R.; Ficha de valoración nutricional del adulto mayor y tabla de valoración nutricional antropométrica según índice masa corporal (IMC) del adultos mayor (>60 años), Ministerio de Salud, Aplicado a los adultos mayores. P.J. La Unión, sector 7, Chimbote, 2012.

\section{DISCUSIÓN}

Tabla 01. Se muestra que del $100 \%$ (171) de los adultos mayores que participaron en el estudio, el 3,5\% (6) tiene un estilo de vida saludable y un 96,5\% (165), no saludable.

Con respecto a lo investigado se aprecia un menor porcentaje de adultos mayores con un estilo de vida saludable, ya que son pocos los adultos mayores que toman importancia sobre el mantenimiento de su salud, acudiendo al puesto de salud o buscando ayuda para su bienestar, para informarse sobre lo que deben realizar para mantener un buen estado de salud. Además, hace falta más trabajo preventivo promocional e importancia del programa de adulto mayor de parte del establecimiento de la jurisdicción.

En cuanto a su alimentación, manifiestan no comer a la hora e incluso, no consumen alimentos balanceados o saludables; tampoco acuden al puesto de salud a realizarse chequeos médicos. 
En ese sentido, el estilo de vida es la manera en que vive una persona (o un grupo de personas). Se basa en patrones de comportamientos identificables, determinados por la interacción entre las características personales e individuales, las interacciones sociales y las condiciones de vida. Una forma de vida también refleja las actividades, los valores o la visión del mundo en un individuo. ${ }^{3}$

El estilo de vida es el conjunto de actitudes y comportamientos que adoptan y desarrollan las personas de forma individual o colectiva para satisfacer sus necesidades como seres humanos y alcanzar su desarrollo personal, teniendo repercusión en la salud tanto física como psíquica. Un estilo de vida saludable repercute en forma positiva en la salud. Comprende hábitos como la práctica habitual de ejercicios, alimentación adecuada y saludable, el disfrute del tiempo libre, actividades de socialización, mantenimiento de la autoestima alta, etc. Un estilo de vida poco saludable es causa de numerosas enfermedades, como la obesidad o el estrés. Comprende hábitos como el consumo de sustancias toxicas (alcohol, drogas), tabaquismo, sedentarismo, prisas, exposición a contaminantes. ${ }^{4}$

Estilo de vida, hábito de vida o forma de vida hace referencia a un conjunto de comportamientos o actitudes cotidianos que realizan las personas, algunas de las cuales pueden ser no saludables. Los estilos de vida están determinados por los procesos sociales, las tradiciones, hábitos, conductas y comportamientos de los individuos y grupos de población que conllevan a la satisfacción de las necesidades humanas para alcanzar la calidad de vida. ${ }^{5}$

De igual modo, el estilo de vida es la base de la calidad de vida, concepto que la Organización Mundial de la Salud (OMS) define como "la percepción que un individuo tiene de su lugar en la existencia, en el contexto de la cultura y del sistema de valores en los que vive y en relación con sus objetivos, sus expectativas, sus normas, sus inquietudes". ${ }^{6}$

En el presente estudio podemos observar que la mayoría de los adultos mayores del Pueblo Joven La Unión, sector 7 presenta un estilo de vida no saludable, lo cual nos da a entender que la población mantiene quizás hábitos y costumbres no saludables, como son alimentación inadecuada, ya sea por desconocimiento o quizá también por la precaria situación económica en la que se vive, así como también podríamos decir por la falta de oportunidades de trabajo.

Los estilos de vida poco saludables, son aquellos que aumentan las posibilidades de que una persona desarrolle una enfermedad específica. Algunos factores de riesgo incluyen edad, estilo de vida (fumar, por ejemplo), antecedentes médicos familiares y enfermedades previas. Una forma de vida rutinaria también refleja las actitudes, valores o la visión del mundo de un individuo. ${ }^{7}$ 
Tabla 02. Se muestra la distribución porcentual del estado nutricional de los adultos mayores que residen en el P.J. La Unión. Probablemente los resultados encontrados se deben a que investigan a los adultos mayores que pertenecen a instituciones donde el personal de salud les provee de cuidados necesarios y se preocupan de su estado nutricional, encontrando cifras altas de normopeso; sin embargo, en estos lugares puede haber sobrepoblación de adultos mayores, lo que dificulta su atención; además, el adulto mayor alejado de sus familiares puede presentar problemas de ansiedad, dando como consecuencia delgadez, sobrepeso y obesidad.

Asimismo, los resultados encontrados posiblemente se presenten debido a que el adulto mayor no conoce o demuestra poco interés acerca de una alimentación adecuada para su etapa de vida; no tienen apoyo de sus familiares y solo consumen de lo que disponen; algunos tienen posibilidades de alimentarse mejor, pero por ignorancia consideran que como ya son ancianos solo deben comer lo necesario; además, muy pocos realizan actividad y ejercicio físico; solo se dedican a su hogar y al cuidado de los nietos.

Por otro lado, existen adultos mayores que presentan delgadez debido a que solo consumen dos comidas al día, no tienen una rutina de alimentación, o llegan a consumir alimentos cuando sus familiares llegan del trabajo, sin contar con los que viven solos, causando alteraciones del aspecto emocional, como depresión, ansiedad, aislamiento, entre otros aspectos.

Los resultados obtenidos se deben a una combinación entre factores culturales, físicos y emocionales, tales como el abandono familiar y la depresión, llegando a no comer, como una forma de protestar o de llamar la atención, así como también la ingesta exagerada, producto de estados ansiosos o de soledad. Es bien sabido que el estado emocional influye en el estado físico, sobre todo este grupo etario.

Todas estas razones producen en el adulto mayor del P.J. La Unión, sector 7, un estado nutricional inadecuado, lo que los hace más sensibles a los problemas de salud en el futuro, tales como problemas cardiovasculares (hipertensión, etc.), debido a que los adultos mayores con sobrepeso (aproximadamente la cuarta parte), tienen una alimentación no balanceada, llegando a consumir en su mayoría alimentos ricos en grasas y carbohidratos, por lo que se necesita mayor promoción en este aspecto de parte del establecimiento de la jurisdicción.

Existe un sinnúmero de factores de riesgo que facilitan o provocan malnutrición en el senescente, entre las que se pueden destacar la falta de educación nutricional, ingesta inapropiada de alimentos (cantidad y/o calidad), pobreza, aislamiento social, dependencia y/o discapacidad, patologías asociadas (agudas 
y /o crónicas), tratamientos farmacológicos, edad avanzada, trastornos afectivos y cognitivos. ${ }^{8}$

Un estado nutricional alterado constituye un factor de riesgo que se asocia a numerosas enfermedades crónicas y deteriora el pronóstico en patologías agudas. En los senescentes existe una relación recíproca entre nutrición y enfermedad; así por ejemplo, se enferman más los ancianos desnutridos y se desnutren más los ancianos enfermos. Se ha reportado una elevada prevalencia de las alteraciones del estado de nutrición en los adultos mayores (desnutrición y obesidad) y una mayor morbilidad y mortalidad asociadas a dichas alteraciones. ${ }^{9}$

Además, la Organización Mundial de la Salud (OMS), en el estudio de nutrición en el envejecimiento, señaló a la población anciana como un grupo nutricionalmente muy vulnerable, debido a los cambios anatómicos y fisiológicos asociados al proceso de envejecimiento. ${ }^{10}$

Los resultados presentados en la investigación demuestran que menos de la mitad de los adultos mayores tienen un estado nutricional normal, ya que los adultos consumen en sus comidas diarias alimentos variados como vegetales, frutas, carnes, legumbres, cereales, granos y productos lácteos; mientras que en menor porcentaje muestra que consumen alimentos balanceados; además no consumen la última comida (cena), entre otros aspectos; ingieren alimentos con alto contenido de grasa y harinas, lo que conlleva a una alteración nutricional.

Los conocimientos sobre nutrición en el área de salud es una responsabilidad que toda la población en general debe mantener como cultura general. Se debe orientar a las personas adultas sobre los tipos de dietas balanceadas en adultos mayores. La población de La Unión, sector 7, no tiene conocimientos sobre una dieta específica para su salud, lo cual lleva a la población adulta a ser vulnerable a contraer enfermedades.

Tabla 03. Se evidencia que la mayoría de los adultos mayores tiene estilo de vida no saludable, prevaleciendo el estado nutricional normal $(37,4 \%)$ del total, siendo mayor respecto al estado nutricional alterado el sobrepeso $(26,9 \%)$; inmediatamente se encuentra la delgadez (24\%) y, en última instancia, la obesidad $(8,2 \%)$ del total. Los resultados no muestran prueba chicuadrado porque una categoría del estilo de vida tiene un porcentaje pequeño.

Los adultos mayores del P.J. La Unión, sector 7, en su totalidad presentan estilo de vida no saludable y un mínimo porcentaje estilo saludable; sin embargo, se observan cifras altas de estado nutricional normal, lo que hace pensar que existen otros factores que participan y que influyen en su estado nutricional. Probablemente muchos de ellos consumen una dieta rica en carbohidratos y 
tienen una vida sedentaria, lo que haría pensar en un estado de sobrepeso u obesidad; sin embargo, presentan un estado nutricional normal. Es que en el adulto mayor la fisiología funciona de manera diferente y actúa en él muchos factores como la lentitud de la digestión y la dificultad en la absorción de los alimentos.

Los adultos mayores del P.J. La Unión, sector 7, llevan un estilo de vida no saludable en su mayoría, no se alimentan bien, no realizan ejercicios, no hay recreación, etc.

Un estilo de vida físicamente activo, con abstención del consumo de tabaco, consumo moderado de alcohol y la elección de alimentos saludables, maximizan las posibilidades de tener un peso dentro de lo considerado normal. ${ }^{11}$

El estilo de vida de una persona está compuesto por dimensiones y las personas deciden de acuerdo a su contexto si adoptan esas dimensiones saludablemente o no. En la investigación se encuentran resultados de elevados porcentajes de un estado nutricional normal en los adultos mayores; sin embargo, con estilo de vida no saludable.

Asimismo, cifras altas presentan sobrepeso y obesidad, probablemente por llevar una vida sedentaria. No realizan ejercicios de manera regular. Tienen la idea de que al llegar a la vejez el adulto debe realizar poca actividad. Se observa que ese grupo etario solo se dedica al cuidado de la vivienda, mientras que los demás miembros laboran y otros se dedican al cuidado de los nietos. Además, muchos de ellos están acostumbrados a consumir una alimentación principalmente en base a harina.

Martínez J. y Serra LL. ${ }^{12}$, en su tesis doctoral, analizando la dieta y la actividad física como determinantes del estado nutricional en mujeres sanas, con edades comprendidas entre los 60 y los 80 años, detectó marcadas diferencias de peso entre las mujeres sedentarias y las mujeres con una elevada actividad física y llegó a la conclusión de que si las mujeres presentaban elevado peso corporal se debía al descenso de la actividad física producida; por tanto repercutía en su estado nutricional y en su forma de vida (estilo de vida).

A pesar de que no se trabajó en el presente estudio con dimensiones, en los estudios antes mencionados se hace evidente que la actividad física influye en el estado nutricional de los adultos mayores: aquellos que no hacen ejercicios presentan sobrepeso y obesidad, aunque hay que tener en cuenta que en este grupo etario el funcionamiento del organismo es diferente y no solo actúa en él la alimentación, sino otros factores como el metabolismo.

Los resultados de cifras altas de estado nutricional normal y sobrepeso se presentan en adultos mayores que se encuentran entre los 60 a 64 años de edad y 
comen tres veces al día y no realizan actividad física; sin embargo, los de bajo peso (delgadez) presentan una edad mayor y se alimentan entre una y dos veces al día, atribuible esto último fundamentalmente al poco acceso a los alimentos, el mito de "que comer de noche hace daño" y el no gustarles determinados alimentos.

En los adultos mayores hay una disminución de la intensidad de la actividad física realizada, así como el tiempo dedicado para esta, lo que corresponde a un incremento de peso y adiposidad en una primera etapa y posteriormente deterioro de la muscularidad con pérdida ponderal. El metabolismo en reposo disminuye con la edad, justifica la pérdida de masa muscular activa y el incremento paralelo de los depósitos de grasa que son metabólicamente inertes. ${ }^{13}$

Por otro lado, el estado emocional de los adultos mayores también podría ser una causa del estado nutricional que presentan, ya que en el P.J. La Unión, sector 7, prácticamente se encuentran en situación de abandono y esto hace que surja en ellos sentimientos de soledad y desamparo.

Otros aspectos, como la soledad, el aislamiento familiar, la falta de cuidado y de redes de apoyo, conducen en muchos casos a la depresión. Esta es una patología más prevalente en este grupo de población de lo que se diagnostica. Los viejos se van quedando solos, sus amigos y familiares van muriendo, tienen miedo a la soledad, a la indefensión y al desamparo. ${ }^{10}$

Según García E. ${ }^{14}$, aspectos como los mencionados, que se refieren a la disminución del consumo de energía, descenso de la actividad física, disminución de la tasa metabólica basal, enfermedades crónicas, desnutrición, disminución de la fuerza y la resistencia, hacen parte del denominado ciclo de la fragilización, aparte de otros factores, como los genéticos, la enfermedad, el envejecimiento y factores relacionados con los estilos de vida que generan alteraciones en el estado nutricional que contribuyen a la disminución de la reserva funcional y la capacidad de respuesta de los individuos a factores exógenos o endógenos que generen estrés.

Los adultos mayores del P.J. La Unión, sector 7, presentan un estado nutricional normal con estilo de vida no saludable, debido a que, entre semana, consumen frutas y verduras y por el proceso fisiológico que presentan. Además, los adultos mayores que presentan delgadez, probablemente están acostumbrados a consumir dos veces al día comida, no tienen apoyo de sus familiares y el personal del establecimiento de salud no los visitan. Muchos de ellos presentan abandono, causando depresión, ansiedad y, como consecuencia, disminución del apetito; además, algunos adultos mayores presentan sobrepeso, delgadez y obesidad. Posiblemente, el factor causal es la falta de educación 
nutrición, ingesta inapropiada de alimentos (cantidad y/o calidad), pobreza y aislamiento social.

Los resultados de la tercera tabla nos muestran que la mayoría de adultos mayores tienen un estilo de vida no saludable y un mínimo porcentaje, estilo saludable; sin embargo, existen elevados porcentajes de un estado nutricional de sobrepeso, delgadez y obesidad, lo que estaría indicando la necesidad de trabajar en el cambio del estilo de vida del adulto mayor.

\section{CONCLUSIONES}

La mayoría de adultos mayores del P.J. La Unión, sector 7, presenta un estilo de vida no saludable y un mínimo porcentaje, estilo saludable.

En cuanto al estado nutricional de los adultos mayores, cerca de la mitad de los adultos mayores presenta un estado nutricional normal; casi la cuarta parte presenta sobrepeso; un porcentaje menor presenta delgadez y una mínima parte, obesidad.

Los adultos mayores que tienen un estilo de vida no saludable presentan en mayor porcentaje estado nutricional normal, sobrepeso, delgadez y obesidad, respectivamente, y los adultos con estilo de vida saludable presentan en mínimo porcentaje obesidad, sobrepeso, normal y delgadez, respectivamente.

\section{REFERENCIAS BIBLIOGRÁFICAS}

1 Gómez J, Jurado M, Viana B, Da M, Hernández A. Estilos y calidad de vida. [Revista digital en internet]. Buenos Aires; 2005 [Citado 2012 Nov. 28]. [Alrededor de 10 pantallas].

Disponible desde URL: http://www.efdeportes.com/efd90/estilos.htm.

2 MinisTERIO DE SALUD. Alimentación y nutrición saludable en la etapa del adulto mayor de 60 a más años. Lima-Perú. [Citado 2012 Nov. 28]. [Alrededor de 19 pantallas]. Disponible desde URL: fttp://ftp2.minsa.gob.pe/descargas/dgps/compendio/pdf/102.pdf.

3 HeRnÁNDEZ, R. Metodología de la Investigación. 3 ed. Editorial Mc Graw Hill. Interamericana México 2003. Consulta 26 de noviembre del 2008. Disponible en: http://www.metabase.net/ docs/unibe/03624.html.

4 Schoenbach V. Diseños de estudio analítico. [Monografía en internet]. 2004. [Citado 2012 Dic. 19]. [44 páginas]. Disponible en URL:http://www.epidemiolog.net/es/endesarrollo/DisenosDeEstudio Analiticos.pdf

5 Dionisio F, ZALdíVAR P. Prevención del estrés en la tercera edad. [Monografía en internet].Cuba 2004. [Citado 2012 Dic. 19] [Alrededor de 02 pantallas]. Disponible en URL: http://www.sld.cu/saludvida/ adultomayor/temas.php?idv $=5940$.

6 CARrasco V. Malnutrición en el anciano [Articulo en internet]. Madrid- España 2006. [Citado 2012 Nov. 28]. [Alrededor de 02 pantallas]. Disponible desde URL: http://bvs.Insp.mx/artículos/5/2/ 092001.htm

7 MuÑOZ S Y CRIBILLERO V “Estilo de vida y estado nutricional en el adulto mayor en el A.H. Esperanza Baja- Chimbote, 2010. [Tesis para obtener el título de licenciada en enfermería]. Universidad católica de los Ángeles de Chimbote 2010. 
8 REYES L y ROMERo M. En su estudio Estilo de vida y factores biosocioculturales de la persona en la comunidad de Sallalli. Ayacucho, 2009. [tesis para optar el grado de licenciada en enfermería]. Ayacucho 2009.

9 FELIPE L. "Estilo de vida y factores biosocioculturales del adulto mayor A.H. Villa Magisterial I y II Etapa, Nuevo Chimbote, 2010", [Tesis para optar el Titulo de Licenciada en Enfermería]. Chimbote: Universidad Católica Los Ángeles de Chimbote, Facultad de Enfermería; 2010.

10 CARrión L. Estilo de vida y factores biosocioculturales de la mujer adulta mayor, A.H. 3 de OctubreNuevo Chimbote. [Tesis para optar por el Titulo de Licenciada en Enfermería]. Chimbote: Universidad Católica los Ángeles de Chimbote. Facultad de Enfermería, 2011.

11 FERNÁNDEZ, Y. Ritmos populares cubanos como una opción para mejorar el estilo de vida. [documento en Internet]. Cuba [Citado 2013 Sep. 28.] disponible en URL: http://www.efdeportes.com/efd149/ sanabanda-ritmos-popularescubanos-para-amas-de-casa.htm

12 MARTínez J y SERRA LL. Hábitos alimentarios y actividad física en el tiempo libre de las mujeres adultas catalanas. [Tesis doctoral]. Universidad de Barcelona. [Citado 2013 Sep. 28]. Disponible desde URL: http://www.tdr.cesca.es/TESIS_UB/AVAILABLE/TDX-0314105-115414/1.Introducc\%F3n_ y_objetivos.pdf.

13 Eva M. Estilo de vida [serie en internet]. 2009 [citado 2013 junio 20]: [Alrededor de 1 pantallas]. Disponible desde el URL: http://www.consumoteca.com/diccionario/estilo-de-vida

14 García E. La participación del estado nutricional en la fragilizarían del individuo. Rev. Nut clín. [Citado 2013 Sep. 28]. [alrededor de 20 pantallas]. Disponible desde URL: http://www.amaee.org.mx/ docs/revnutcli/Nutricion-1-03.pdf

15 Estilo de vida saludable [serie de internet]. 2012 [citado 2013 junio 20]: [Alrededor de 1 pantalla]. Disponible en el URL: http://www.uniminuto.edu/web/uvd/-/estilos-de-vida-saludables

16 Solano y Colab. Encuesta de Universidad ICESI. 2004. [Citado 2011 Jul. 13]. [5páginas]. Disponible desde el URL: http://www.DEFINICION + DE +ESTILO +DE + VIDA + POCO + SALUDBLE\&cd = $11 \& \mathrm{hl}=\mathrm{es} \& \mathrm{ct}=\mathrm{clnk} \& \mathrm{gl}=\mathrm{pe}$

17 ARMAS C. Y LópeZ Z. En su estudio Estilo de vida y estado nutricional del adulto mayor de la Urbanización Bruces. Nuevo Chimbote, 2010. [Tesis para optar el título de licenciada en enfermería]. Nuevo Chimbote, Universidad Católica los Ángeles de Chimbote. Facultad de Enfermería; 2010.

18 LozAno D. y SAAVEDRA S. En su estudio Estilo de vida y estado nutricional del adulto mayor. A.H. Ramal Playa, Chimbote, 2012. [Tesis para optar el título de licenciada en enfermería]. Chimbote, Universidad Católica los Ángeles de Chimbote. Facultad de Enfermería; 2010.

19 CABRERA Y. Evaluación antropométrica de las personas aisladas en el ancianito Daniel Álvarez Sánchez durante junio del 2008 [monografía en internet] [Citado 2013 Sep. 28] [alrededor de 5 pantallas] disponible desde URL: http://slideshare.net/pvgonzalez/evaluacion-antropometricaen-el-ancianatodaniel-alvarez-sanchez.

20 CERECEDA M. Antropometría nutricional de adultos mayores. CAM-Fiori. C.I Bioquímica y Nutrición. An. Fac. Med. [revistas de trabajos clínicos] [Citado 2013 Sep. 28]. 64 (3): 28- 58; Lima- Perú: 2003 disponible. Desde URL: http://sisbib.unmsm.edu.pe/bvrevistas/canales/v64_sup/trabajo_clini.htm.

21 SEdó P. La Atención Nutricional- Adultos Mayores formato de archivo: PDF/Adobe Acrobat de MSPS Masís- Artículos relacionados a la orientación nutricional del adulto mayor necesariamente desde estar involucrada su familia. San José [Tesis para optar el título de Licenciada en Nutrición] [Alrededor de 10 pantallas]. Disponible URL: www.redadultosmayores.comar/buscador/files/SALUD021.PDF.

22 Tocón R. Valoración del anciano en la actualidad actual. GEROKOMOS. En España, 2000; [Citado 2013 Sep. 28]. [Alrededor de 06 pantallas]. Disponible desde URL: http://www.scielo.cl/scielo.php? pid $=$ S071775182006000500006\&script $=$ sci_arttext 